\title{
Skin microvascular morphology and hemodynamics during treatment with veno-arterial extra-corporeal membrane oxygenation
}

\author{
Torjus Wester ${ }^{\mathrm{a}, \mathrm{b}, *, 1}$, Zaheer Ahmed Awan ${ }^{\mathrm{a}, \mathrm{b}}$, Tobias Skylstad Kvernebo ${ }^{\mathrm{a}, \mathrm{c}}$, Göran Salerud ${ }^{\mathrm{d}}$ \\ and Knut Kvernebo ${ }^{\text {a,b }}$ \\ ${ }^{a}$ Circulation laboratory, Department of Cardio-thoracic Surgery, Oslo University Hospital, \\ Ullevaal, Norway \\ ${ }^{\mathrm{b}}$ Medical Faculty, University of Oslo, Norway \\ ${ }^{\mathrm{c}}$ Volvat Medical Centre, Oslo, Norway \\ ${ }^{\mathrm{d}}$ Institute of Biomedical Engineering, Linköping Univeristy, Sweden
}

\begin{abstract}
.
OBJECTIVES: To explore the potentials of microcirculatory assessments for predicting outcome of patients treated with extra corporeal membrane oxygenation for cardiogenic shock.

METHODS: Eight patients with acute cardiogenic shock treated with ECMO and eight healthy controls were examined with skin vital microscopy and laser Doppler perfusion measurements.

RESULTS: Three patients died on ECMO (group 1). Five patients were successfully weaned off ECMO (group 2). Four patients were discharged from hospital and one died after successful weaning from bleeding complications. Patients surviving ECMO (group 2) had microcirculatory findings comparable with healthy controls. Patients in group 1 showed major skin microvascular pathology: pericapillary bleedings $(n=1)$, pericapillary dark haloes $(n=2)$ and capillary micro thrombi $(n=1)$. As compared with survivors they had lower functional capillary density (FCD) $(n=3)$, higher heterogeneity of functional capillary density $(n=3)$ and significantly reduced capillary mean flow-categorical velocity $(n=2)$. Laser Doppler measurements in group 1 had non-significant lower laser Doppler flux values as compared with survivors and controls.

CONCLUSION: Skin microvascular pathology as detected with video microscopy (pericapillary bleedings or haloes, microthrombi/capillaries with "no flow", low FCD with high spatial distribution heterogeneity or low mean flow-categorial velocity) seems to be associated with poor prognosis.
\end{abstract}

Keywords: Capillary flow heterogeneity, capillary pathology, cardiogenic shock, extra-corporeal life support, extra-corporeal membrane oxygenation, microvascular hemodynamics, microvascular monitoring, vital microscopy

\section{Introduction}

Acute cardiac pump failure compromises central hemodynamics and consequently microvascular perfusion throughout the body and the mortality rate varies from 50 to 80 percent [16]. Veno-arterial extra-corporeal membrane oxygenation (va-ECMO) may be used as a bridge to recovery or to other

\footnotetext{
*Corresponding author: Torjus Wester, Oslo University Hospital, Kirkeveien177, 0450 Oslo, Norway. Tel.: +47 22119134 ; Fax: +47 221174 70; E-mail: torjus.wester@medisin.uio.no.

${ }^{1}$ Present address: Department of Plastic and Recontructive surgery, Oslo University Hospital.
} 
destination therapy. Only approximately one-third of adult patients treated on ECMO for cardiogenic shock survive $[5,19]$. Mortality is most often caused by sepsis, multi organ failure or bleeding complications. ECMO treatment is resource demanding and in a recent editorial Hermansen et al. state that: "The assumption that improved technological solutions and hemodynamics translates into improved survival may not be completely valid" [15].

To survive and function, cells are dependent on oxygen supply and nutrition supplied by the microcirculation. Several non-invasive microvascular techniques have been developed and improved for clinical use during the last decades and different vascular beds response to physiologic/pathologic conditions have been examined $[4,13,14,29]$. Even though microcirculatory targets may represent the most important endpoints to optimize therapy of circulatory shock, microvascular examinations are hardly used routinely in any clinical discipline [24].

The present study was undertaken in a medium sized cardiac surgical unit. We examined skin microvascular morphology and perfusion in patients treated with ECMO following acute cardiac pump failure. The hypothesis was that demonstration of microvascular pathology soon after establishment of ECMO, imply reduced chance of survival.

\section{Patients and methods}

\subsection{Patients}

Over a period of two years, patients treated with ECMO for cardiogenic shock in our medium sized cardiac surgical unit (approximately 550 open heart operations per year) were candidates for inclusion. Eight consecutive patients (E1-E8), two females and six males; median age 59 years (range 27-78), were included. No patients were excluded from the study. Four patients (E4-E7) were in a state of cardiogenic shock before reaching the operating theatre: one secondary to massive pulmonary embolism with ongoing mechanical cardiac compression (E4), one with massive myocardial infarction (MI) (E5), one with acute MI and complication to percutaneous coronary intervention (PCI) (E6) and finally one with endocarditis (E7). The remaining four patients suffered from post cardiotomy cardiogenic shock. Eight healthy, non-smoking male students (21-29 years old) served as controls.

\subsection{Extra-corporeal membrane oxygenation (ECMO)}

Arterial cannulation was performed in the groin in seven patients, in two of them via an end to side Dacron graft. One patient had arterial cannulation via the right subclavian artery. Seven patients had vein drainage via the femoral vein and one via the right atrium. The ECMO-circuit consisted of a centrifugal pump (Medtronics Incorporated, Minneapolis, Minnesota, USA), a heparin coated membrane oxygenator and tubes (Maquet Cardiovascular 72145 Hirrlingen, Germany). After establishing ECMO, all patients were initially treated with a flow of at least $4.0 \mathrm{l} / \mathrm{min}$. Maintenance therapy on ECMO was guided by a standard protocol for the unit [33]. Weaning from ECMO was guided by trans-oesophageal ECCO Doppler in addition to clinical parameters.

\subsection{Microvascular techniques}

Skin microcirculation was evaluated with video microscopic measurements in eight patients and Laser Doppler measurements in six. 


\subsubsection{Computer assisted video microscopy (CAVM)}

This technique involves a hand-held video-microscope applied gently on the surface of the region of interest (ROI). Immersion oil is used. Pictures or film sequences are projected and stored on a computer. For the first patient, E1, a less advanced microscope, with a 1.3 megapixel CCD, (ProScan, Bodelin technologies, OR, USA), magnifying 200 times was used. With this microscope pericapillary pathology, functional capillary density (FCD) and heterogeneity could be evaluated, but not capillary flow patterns. The remaining patients (E2-E8) were examined with another microscope (Microvision 2100, Finlay Microvision Co. Ltd., Warwickshire, UK) with higher resolution and a 500 times magnifying lens. An analogue to digital converter (Canopus, Kobe, Japan) was used to project and store the film sequences on a Mac Book pro, using the software iMovie (both Apple, Cupertino, USA).

To study pericapillary pathology, functional capillary density (FCD), heterogeneity of FCD and microvascular flow patterns in a ROI, the first five captured film sequences with adequate quality were used. A grid with four equally sized rectangles was made to facilitate visual analysis [2]. The software Xscope (the Iconfactory, Greensboro, North Carolina, USA) was used for creating the grids. Adequate film quality was defined as focused capillaries in all four quadrants of the frame and sequence duration of at least ten seconds. FCD was defined as the mean number of visible capillaries per square millimetre. Heterogeneity of FCD was expressed as the coefficient of variation ( $\mathrm{CoV}=\mathrm{SD} / \mathrm{mean}$ ) of the density of capillaries in each of the four rectangles in the five film sequences $(n=20)$.

For analysis of flow velocity, each capillary of the five film sequences were visually scored into one of five groups from "no flow" (Category 0) to "brisk flow" (Category 4), and expressed in fractions $(\mathrm{Fr}=$ number of capillaries in each category/total number of capillaries) [32]. Based on the fractions of capillaries in each flow category, mean flow-categorial velocity was calculated in the following way:

Mean flow-categorical velocity $=\{\operatorname{Fr}(1) \times 1\}+\{\operatorname{Fr}(2) \times 2\}+\{\operatorname{Fr}(3) \times 3\}+\{\operatorname{Fr}(4) \times 4\}$.

\subsubsection{Laser doppler perfusion measurements (LDPM)}

LDPM is a technique for quantification of microvascular perfusion. The output of the technique is given in a semi-quantitative scale of flux, defined as the product of number of moving blood cells and their mean velocity in the measured volume $\left(<1 \mathrm{~mm}^{3}\right)$ [34].

A Moor Blood Flow Monitor (MBF 3D) for perfusion measurements with Moorsoft (both Moor instruments, Axminster, Devon, England) was used for recording and analysis. Flux in a ROI was given as the mean value of seven consecutive measurements of a ten-second duration [26].

\subsection{Measuring procedure}

The first microvascular examinations were performed as soon as possible, usually within 24 hours after establishing ECMO, Fig. 1. The second measurement was performed on day 3 when possible. E4 was examined twice with an interval of only two hours before the ECMO was turned off due to ceased cerebral blood flow. Four survivors (Group 2) were controlled 18-65 days after weaning off ECMO. E3 was examined seven times. The skin on the dorsum of the hand, between the first and second metacarpus was examined by CAVM in all patients. From patient 3 and onwards we also assessed the skin perfusion of the medial side of the right foot, with the ROI being located one third of the distance on an imaginary line from the medial malleolus to the caput of the first metatarsus. In the patient with pericapillary bleedings (E1), additional skin areas, such as over arm, thigh, chest, and face were also examined. Six patients were measured by LDPM. Patient E1 was only measured in dorsum of the hand. From patient 4 and onwards LDPM was measured on the foot as well. CAVM and Laser Doppler measurements were performed 


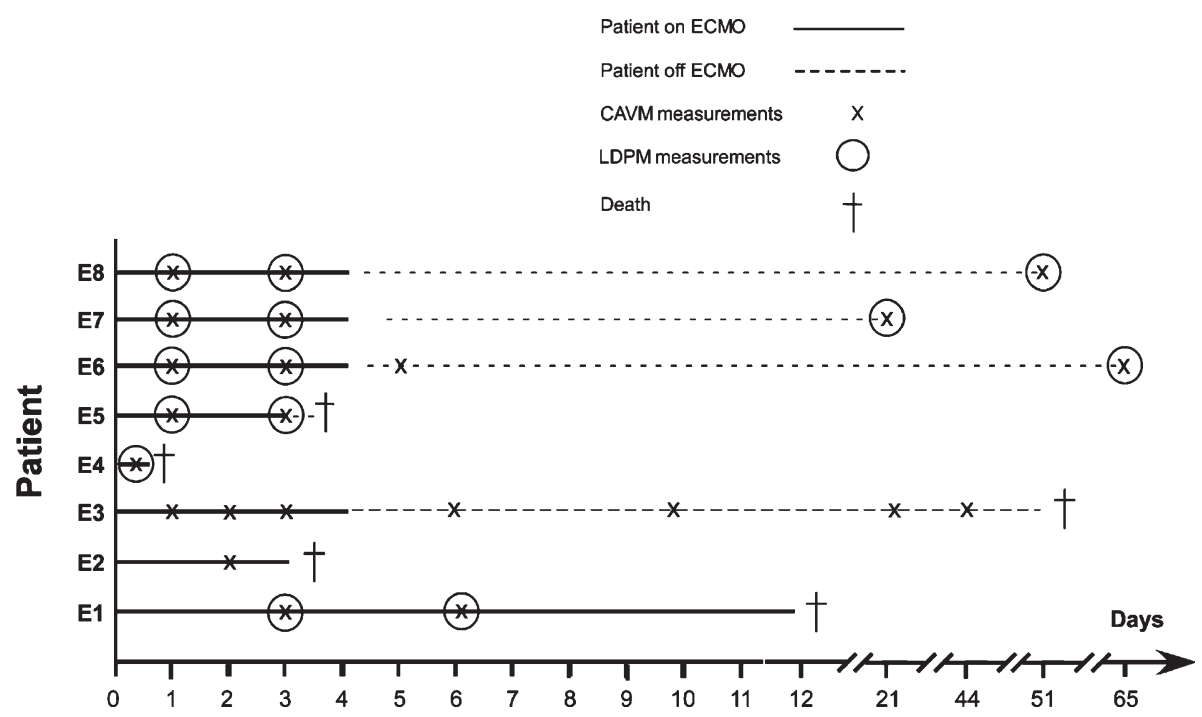

Fig. 1. An overview over the eight ECMO patients, from start of the ECMO treatment till death or last control measurement. Measurement periods are marked according to measuring technique.

in both locations in all controls. At the time of microvascular measurements, the corresponding central hemodynamic parameters, results from blood tests and clinical parameters were noted.

\subsection{Ethical considerations}

The decision to establish ECMO was taken by the responsible surgeon on clinical grounds solely. All microvascular measurements were non-invasive, and performed only after approval from the responsible surgeon and anesthesiologist. No results of microvascular findings influenced maintenance therapy on ECMO.

Since patients on ECMO could not give an informed consent, next of kin, when available, consented to the microvascular assessments. Long-term survivors $(n=3)$ gave their consent to follow-up measurements and to publication of their data. The Regional ethics committee has approved publication of data from all eight patients.

\subsection{Statistics}

Data are presented as means with range. The coefficient of variation $(\mathrm{CoV})$ was used as a parameter for heterogeneity of functional capillary density. For comparing results between outcome groups, independent $t$-test was used.

\section{Results}

\subsection{Clinical information and outcome}

An overview of clinical information of the individual patients, laboratory data, and performed examinations is given in Tables 1, 2 and Fig. 1. 


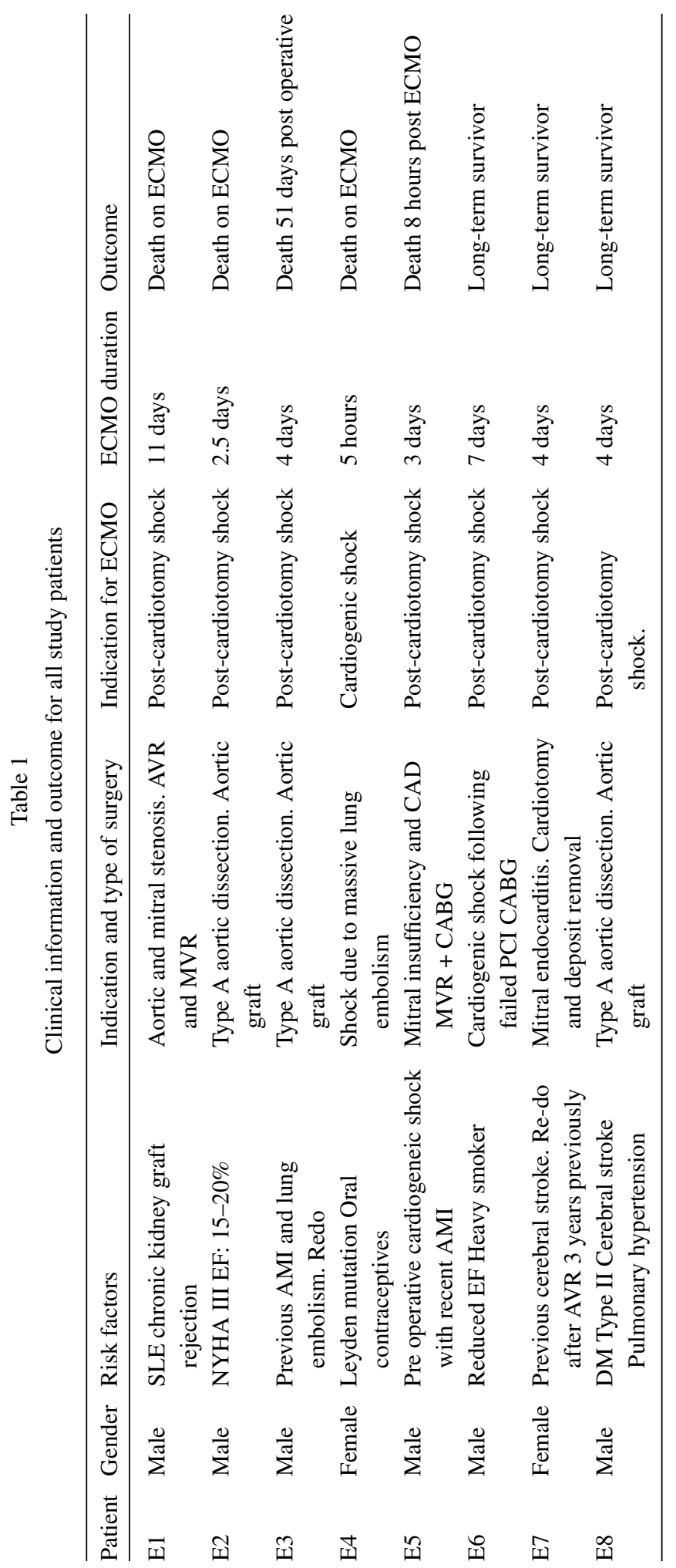


Table 2

Clinical and laboratory data in the ECMO patients at the time of the first microvascular measurement

\begin{tabular}{|c|c|c|c|}
\hline & Group $1(N=3)$ & Group $2(N=5)$ & \\
\hline Hemoglobin (g/dl) & $9.3(7.8-10)$ & $10.1(9.5-11.2)$ & NS \\
\hline Erythrocytes transfusions (no. of units) & $21(10.0-37)$ & $16.4(10.0-20)$ & NS \\
\hline Heart rate (beats/minute) & $74(68-80)$ & $82(55-107)$ & NS \\
\hline MAP (mm Hg) & $53(45-63)$ & $53(45-60)$ & NS \\
\hline CVP (mm Hg) & $19(13.0-28)$ & $10(8-18)$ & NS \\
\hline ECMO (in liters/minute) & $3.5(3-4)$ & $4.1(3.5-4.5)$ & NS \\
\hline Intra Aortic Balloon Pump? & 2 of 3 & 4 of 5 & NS \\
\hline Vasoactive medication? & 3 of 3 & 3 of 5 & NS \\
\hline $\mathrm{FiO}_{2}$ & $0.68(0.45-1.00)$ & $0.60(0.5-0.7)$ & NS \\
\hline $\mathrm{SaO}_{2}$ (in percent) & $86(61-99)$ & $98(98-99)$ & NS \\
\hline $\mathrm{SvO}_{2}$ (in percent) & $70(70$ and 70$)$ & $65.4(55-72)$ & NS \\
\hline Lactate $(\mathrm{mmol} / \mathrm{l})$ & $5.5(1.2-12.5)$ & $3(1.3-3.6)$ & NS \\
\hline $\mathrm{pH}$ & $7.34(7.23-7.41)$ & $7.42(7.36-7.45)$ & NS \\
\hline $\mathrm{pCO}_{2}$ (in $\mathrm{kPa}$ ) & $5.0(4.7-5.4)$ & $4.8(4.4-5.5)$ & NS \\
\hline Base excess & $-4.3(-12.2-(+0.3))$ & $-0.6(-3-(3.5))$ & NS \\
\hline Temperature (in ${ }^{\circ} \mathrm{C}$ ) & $36.8(36.5-37)$ & $37.1(36.9-37.5)$ & NS \\
\hline $\mathrm{CRP}(\mathrm{mg} / \mathrm{l})$ & $77.3(22.0-140.0)$ & $86.5(22.0-173)$ & NS \\
\hline WBC ( $10^{9}$ cells/liter $)$ & $14(12.0-16.0)$ & $7.2(5.0-12.5)$ & $P=0,02$ \\
\hline Urinary output (ml/hour) & $3(0-10)$ & $48(0-100)$ & NS \\
\hline Dialysis (numbers of patients) & 2 of 3 & 1 of 5 & NS \\
\hline Cumulative positive fluid balance (in liters) & $21(8-30)$ & $16.6(10-26)$ & NS \\
\hline
\end{tabular}

The patients were grouped according to clinical outcome into two groups: patients dying on ECMO (Group 1) and patients surviving ECMO (Group 2). Mean age for group 1 was 44 years, for group 2 it was 58 years. Both sexes were present in each group. In group 2, one patient had a recovery of heart function and a maintained cerebral function but died from bleeding complications in the intensive care unit.

Of the remaining four patients in group 2, one patient (E3) was transferred to another hospital where he died from multi organ failure on day 51 after establishing ECMO. The remaining three were still alive and out of institutions two years after the ECMO treatment.

\subsection{Clinical measurements}

White blood cell count, during the first microvascular measurements, was lower among survivors as compared with non-survivors, table 2 .

\subsection{Microvascular measurements}

\subsubsection{Computer assisted video microscopy (CAVM)}

3.3.1.1. Pericapillary pathology. E1 had numerous pericapillary bleedings in several skin locations (dorsum of the hand, volar side of forearm, leg and face) on both examinations, Fig. 2(a). No other patients 


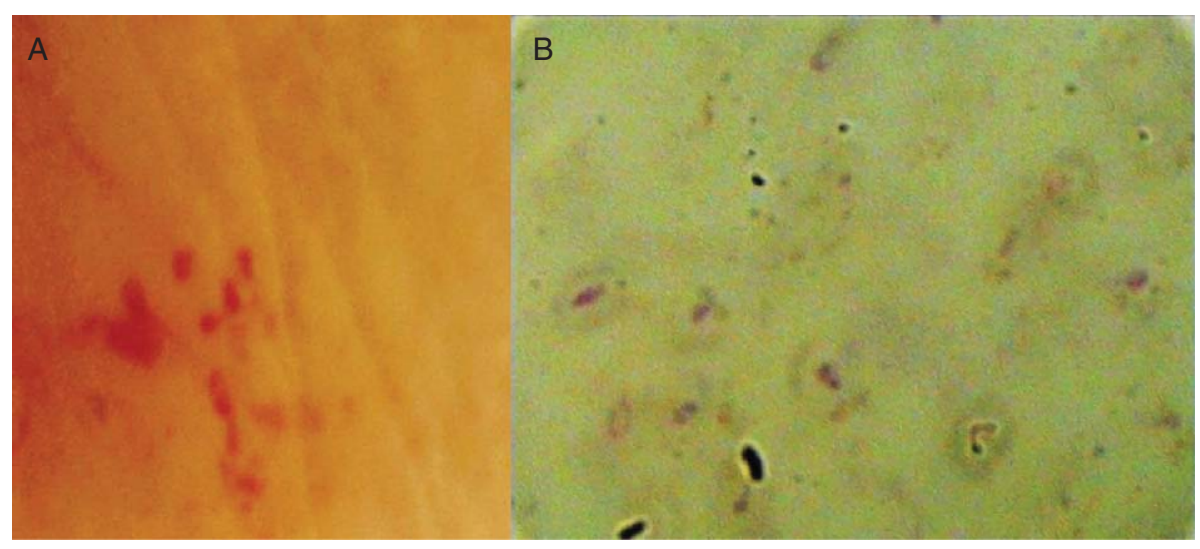

Fig. 2. Skin microcirculation in the dorsum of the hand in two ECMO patients. In A) several pericapillary bleedings are seen, and hardly no dot-liked capillaries are present (Patient E1, Proscan $(\times 200))$. In B) circular haloes are surrounding capillaries with no flow or extremely slow sluggish flow are seen (Patient E2 Microvision $2100(\times 500)$ ).

had any visible pericapillary bleedings. Patients E2 and E4 had circular dark haloes at a distance of $12 \pm 1$ microns around some skin capillaries (65\% in E2 and in 14\% in E4), see Fig. 2(b).

3.3.1.2. Functional capillary density $(F C D)$. The three patients dying on ECMO had significantly lower FCD in the dorsum of the hand compared with patients surviving ECMO $(p=0.002)$, Fig. 3(b). No difference in FCD was seen between patients in group 2 and the controls $(p=0.24)$.

From patient $\mathrm{E} 3$ onwards, measurements were also performed on the medial side of foot $(n=6)$. In this location the patients had an FCD of 66.2 (range 55.6-75.8) capillaries $/ \mathrm{mm}^{2}$, similar to mean FCD of the hand in patients surviving ECMO $\left(70.8\right.$ capillaries $\left./ \mathrm{mm}^{2}\right)$ and controls $\left(65.5\right.$ capillaries $\left./ \mathrm{mm}^{2}\right)$, as well as in the foot of controls $\left(66.2\right.$ capillaries $/ \mathrm{mm}^{2}$ ). Since only one patient in group 1 (E4) was measured on the foot, no further analysis was done on data from this location. Still, E4 had the lowest FCD and the highest $\mathrm{CoV}$ of FCD of the measured patients in this location.

The second measurements of FCD in the hand gave values similar to the first measurements.

Final follow-up measurements of four patients in group 2 (patient E5 had already died) were performed 18-65 days after weaning off ECMO, Figs. 1 and 3. One patient showed a reduced FCD on final assessment (E3, see later), while the rest of the survivors had a mean FCD of 65.5 (range 56.9-76.5) on final assessment, values in the same range as their first measurement and in controls in this location.

3.3.1.3. Heterogeneity of FCD. Patients dying on ECMO (group 1) had significantly higher CoV of skin capillaries compared with patients in group $2(p<0.005)$, Fig. 3(a). No difference was seen between the latter group and controls $(p=0.74)$.

3.3.1.4. Capillary flow patterns. The mean flow-categorial velocity during the first measurements in the skin of the hand showed no difference between patients surviving ECMO, 2.67 (range 2.53-2.87)), and controls with 2.76 (range 2.65-2.88) ( $p=0.17$ ). The two patients that died on ECMO had mean flow velocities of 0.5 and 1.76 , significantly lower than the patients surviving ECMO $(p=0.007)$. In one of these patients with pericapillary dark haloes (E2), erythrocyte movement in the capillaries was hardly detected in any of the film sequences, although the ECMO circuit gave an output of 4.5 liter/min. 

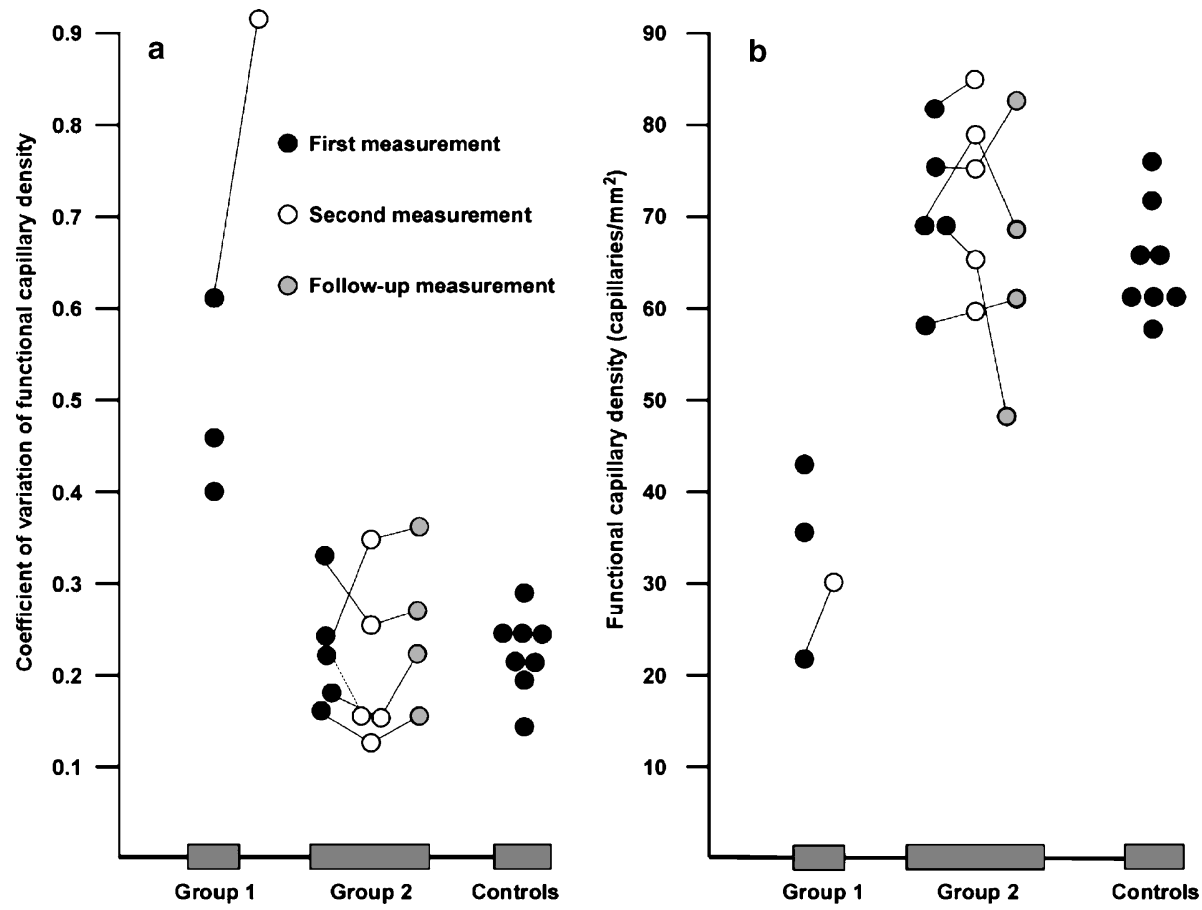

Fig. 3. Functional capillary density (in capillaries $/ \mathrm{mm}^{2}$ ) for three groups of ECMO patients and eight healthy controls (b) and coefficient of variation of functional capillary density for the same patients (a). For patients measured more than once, the first, second and follow-up measurements are given. The follow-up measurements were performed 18-65 days after the patient was successfully weaned off ECMO $(n=4)$.

All capillaries in healthy controls had flow patterns 2 or 3, Fig. 4. Patients in group 2 had a similar flow-category distribution as the controls. In the two patients in group 1 where capillary flow assessments were performed (E2, E4); the capillaries had a broadened and a left-shifted spectrum. Flow category 0 (no flow) and 1 (sluggish flow) was hardly seen in patients surviving ECMO and never seen in controls.

E3 had a prolonged and complicated stay in the ICU with lung infections, sepsis episodes, and progressive renal failure after ECMO treatment. The patient died 51 days after ECMO was established due to multi organ failure. The patient's final microscopy assessments on day 44 showed that FCD was reduced with $30 \%$, CoV was increased with $64 \%$ and the mean flow-categorial velocity was reduced to 1.75 , a reduction of $34 \%$, compared with the initial measurements. On one occasion the patient was examined during an episode with gram negative sepsis (day 24); at that time the FCD was markedly reduced to 49 capillaries $/ \mathrm{mm}^{2}(-29 \%)$, the $\mathrm{CoV}$ was $0.17(-23 \%)$, and $22 \%$ of the skin capillaries had brisk flow (category 4). Capillaries in this flow category were not observed for any other patients at any time.

\subsubsection{Laser Doppler perfusion measurements (LDPM)}

The controls had a mean flux value of $48 \mathrm{Au}$ (range 22-96) in the dorsum of the hand, while the corresponding values at the medial side of the foot were 31.5 (range 17.4-60.1). The mean coefficient of variation was 0.20 (range $0.13-0.35$ ) in the hand, and 0.25 (range $0.12-0.34$ ) at the medial side of the foot. 


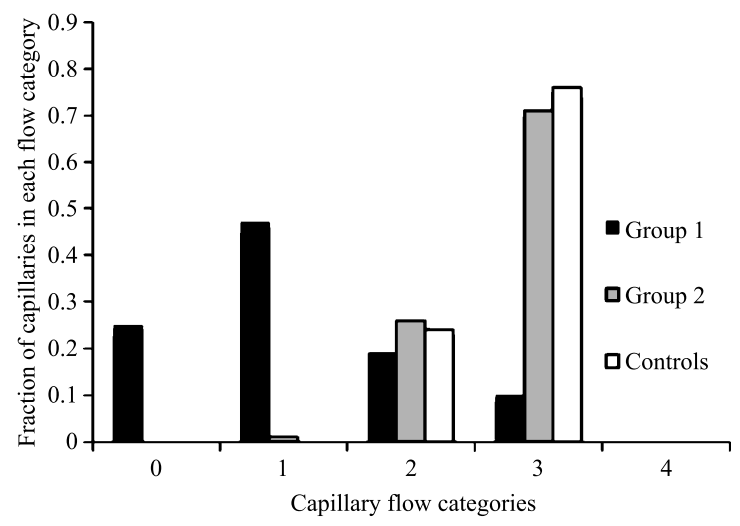

Fig. 4. Distribution of capillaries in each flow category for patients dying on- (group 1), and patients successfully weaned off ECMO (group 2) as compared to eight healthy controls. Flow categories: $0=$ no flow; 1 = sluggish flow (very slow cell movement, sometimes backward flow); 2 = continuous low flow (cells moving continuously forward, mostly slowly); $3=$ continuous high flow (cells moving continuously forward, mostly rapid); 4 = brisk flow (rapidly moving cells throughout the entire film sequence).

Table 3

Laser Doppler flux (in Arbitrary Units) for six ECMO patients.

\begin{tabular}{|c|c|c|c|c|c|c|}
\hline \multirow[t]{2}{*}{ Patient number } & \multicolumn{3}{|c|}{ Dorsum of hand } & \multicolumn{3}{|c|}{ Medial foot } \\
\hline & $\begin{array}{l}\text { 1. Measurement } \\
\text { (Flux/CoV) }\end{array}$ & $\begin{array}{l}\text { 2. Measurement } \\
\text { (Flux/CoV) }\end{array}$ & $\begin{array}{l}\text { 3. Measurement } \\
\text { (Flux/CoV) }\end{array}$ & $\begin{array}{l}\text { 1. Measurement } \\
\text { (Flux/CoV) }\end{array}$ & $\begin{array}{l}\text { 2. Measurement } \\
\text { (Flux/CoV) }\end{array}$ & $\begin{array}{l}\text { 3. Measurement } \\
\text { (Flux/CoV) }\end{array}$ \\
\hline E1 & $4.5(0.31)$ & $4.8(0.18)$ & - & - & - & - \\
\hline E4 & $15(0.45)$ & - & - & $8.0(0.22)$ & $12.4(0.33)$ & - \\
\hline E5 & $299(0.57)$ & $370(0.56)$ & - & $344(0.94)$ & $327(0.26)$ & - \\
\hline E6 & $21.7(0.29)$ & $22.5(0.36)$ & $58.2(0.29)$ & $16.5(0.68)$ & $24(0.48)$ & $29.7(0.11)$ \\
\hline E7 & $74.4(0.37)$ & $40.7(0.29)$ & $76.2(0.17)$ & $30.2(0.13)$ & $24.7(0.28)$ & $82(0.44)$ \\
\hline E8 & $124(0.49)$ & $81.9(0.19)$ & $103.3(0.26)$ & $29.4(0.19)$ & $41(0.22)$ & $23(0.13)$ \\
\hline
\end{tabular}

The laser Doppler data sets for patients are incomplete. The patients that died on ECMO (E1 and E4) had lower flux values in both locations and on both measuring occasions than any of the surviving patients or controls (Table 3), but the differences did not reach significance, probably due to small numbers and large variability.

The coefficient of variation for flux in the skin of the hand was significantly higher in the survivors as compare with the controls at the first measurement $(p=0.03)$, but not for the second and third measurement at the same locations ( $p=0.17$ and 0.74 respectively). On the medial side of the foot, coefficient of variation did not show significant changes between survivors and controls ( $p=0.22,0.44$ and 0.82 respectively).

\section{Discussion}

The study was undertaken in a medium sized unit for heart surgery, with approximately 550 open heart operations a year. Both the incidence of patients in need of ECMO $(0.6 \%)$ and survival of patients treated with ECMO (28\%) are comparable with data from the literature [5, 19]. Except for white blood 
cell count no clinical parameters showed significant differences between the survivors and non-survivors. Significant changes were seen for all parameters of CAVM. Skin microvascular anatomy is complex with subpapillary capillaries mainly serving nutrition for epithelial proliferation and deeper vascular plexus mainly serve body temperature regulation. In adult skin, only the sub-papillary capillaries are seen in most locations with the microscopy equipment used in this study. The Laser Doppler techniques measure both superficial and the deeper plexus perfusion.

\subsection{Observations made with computer assisted video microscopy (CAVM)}

\subsubsection{Functional capillary density and heterogeneity of capillary distribution}

In 1920 the Danish physiologist August Krogh was awarded the Nobel Prize in Physiology or Medicine. One of his main achievements was the identification of the "Krogh cylinder", postulating that all cells need to be located within a critical radius of a perfused capillary to survive [21]. Cells outside this radius would experience insufficient oxygenation independent of the flow rate and erythrocyte oxygen saturation in the nearest capillary. An uneven distribution of perfused capillaries may give low oxygen tension to some cells in spite of normal $\mathrm{SaO}_{2}$.

The patients that died on ECMO had reduced FCD and increased CoV of FCD compared with patients surviving ECMO and healthy controls. The patients surviving ECMO had stable FCD and CoV within the reference levels for the controls (Fig. 3). The exception was patient E3, who had a complicated post ECMO course with several septic episodes. The last assessments, seven days before he died from multi organ failure, showed values comparable to the patients dying on ECMO see Fig. 3.

In 1922 Freedlander used a microscope to show a decreased capillary density in skin in septic patients [12]. Later studies confirmed Freedlanders findings and demonstrated increased heterogeneity of FCD in different tissues and mammalian species with systemic diseases [6, 7, 9, 23, 28, 30, 31]. In patients with septic and cardiogenic shock a persistent severely reduced FCD for 24 hours in the sublingual area, is associated with increased mortality [6,7]. Reduced FCD in the rectal mucosa is associated with poor prognosis in patients with severe malaria [9].

\subsubsection{Capillary flow-patterns}

In the two patients in group 1, in whom capillary flow had been analysed, a significant number of capillaries with "no flow" or "sluggish flow" were seen. These categories were hardly seen in controls or survivors. Patients in group 2 had capillary flow pattern similar to the controls. An increased number of no-flow capillaries have been described in different diseases and a positive correlation between capillaries with no-flow and high mortality has been shown $[6,7,9]$. All capillaries in patient E2 had flow category 0 or 1 , fifty per cent in each category. Interestingly the patient at that point had biochemical markers indicating disseminated intravascular coagulation (DIC).

\subsubsection{Circular dark haloes}

Dark haloes were found in two of the patients who died on ECMO (E2 and E4), with the halo edges $12 \pm 1$ microns from the capillaries. In E2 numerous haloes were present, prominently around capillaries with "no flow". E4 had fewer haloes, and they were also seen surrounding perfused capillaries. The cause of these haloes is uncertain, but one possibility is that they represent precipitated proteins or erythrocyte degradation products leaking from injured capillaries. Another possibility is that they are caused by pericapillary edema. Bollinger has described similar dark haloes in the lower extremity of patients with chronic venous insufficiency [3]. 


\subsubsection{Pericapillary bleedings}

Bleeding capillaries have been described in patients with von Willebrand disease, in patients with critical lower limb ischemia, in patients with connective tissue disorders and in patients on anticoagulants [1, $10,20,22,25]$. We have reported pericapillary bleedings in the tongue of septic pigs four hours after injection of $N$. meningitides antigen [32]. Pericapillary bleedings was found in several skin locations of patient E1 (died on ECMO). This patient suffered from systemic lupus erythematosus. He had not been treated with anticoagulants. No macroscopic bleedings could be detected. Capillary erythrocyte leakage is the result of severe damage to the capillary wall and increased fluid leak and edema would be expected. Patient E1 gained 30 kilos body weight during the first 24 hours on ECMO, and gained another 12 kilos between the first and second measurement.

\subsection{Laser Doppler perfusion measurements}

The major part of skin perfusion takes place in the deeper thermoregulatory plexuses where perfusion is mainly regulated by sympathetic activity. Since perfusion in these plexuses mainly serve a thermoregulatory function, skin nutrition can not be assessed by the LDPM technique [28]. Even though the two patients that died on ECMO (E1 and E4) had the lowest perfusion values of all in both locations and measuring periods, no significant differences were demonstrated due to small numbers and large data variation.

\subsection{Limitations}

The number of included patients is small and represents a heterogeneous group with acute heart failure, while the reference data were collected from healthy young male students. Since capillary erythrocyte velocities in healthy subjects are not age dependent, we assume that our control group of young students can be used [18].

\subsection{Possible implications of the findings}

In USA, $40 \%$ of the Medicare expenditures occurred in the last month of life and in-patient expenses accounted for over $70 \%$ of the decedents' total costs [27]. This indicates increased use of high-tech intensive care facilities when approaching the end of the patients' lives, without gaining much improvement of life expectancy. ECMO and other circulation assist devices are costly in use. Generally accepted criteria for selection of patients for such treatment are missing. Assist devices improve central hemodynamics, but often without improving life expectancy [11]. It is therefore a strong need for diagnostic techniques that can be used to select patients for expensive extracorporeal life support techniques, for estimation of prognosis early after establishing such treatment and to assess the effects of supportive treatment during the use of an assist device.

The idea that the microscopic examination of sublingual microcirculation may serve as a prognostic indicator of critically ill patients with sepsis or cardiogenic shock seems to be accepted [6-8]. In a study on 68 patients with cardiogenic shock, reduced sublingual functional capillary density was associated with development of organ failure [31].

A case report on one patient on ECMO examined sublingually by a microscopic method (OPS) showed that capillary flow velocity varied with varying ECMO flow. The changes were most prominent in the smallest capillaries [17]. The small sublingual capillaries correspond to the size of the nutritive skin papillary capillaries examined in our study. 
Our study indicates that techniques for bedside assessments of skin microcirculation can be developed to valuable clinical tools for improved handling of patients on assist devises.

\section{Conclusion}

Microvascular examinations of skin nutritive capillaries in patients on ECMO show major structural and functional pathology in patients dying on ECMO, while patients surviving ECMO have results similar to healthy controls. We believe the finding of intact skin microcirculatory morphology and function in survivors early after establishment of ECMO is a robust and clinically useful finding implying a good prognosis. Pericapillary bleedings or dark haloes, micro-thrombi/capillaries with "no flow", low capillary flow velocity and low functional capillary density are associated with poor prognosis.

\section{Acknowledgments}

Statistical advice was given by professor, Leiv Sandvik, University of Oslo.

\section{Conflicts of interest}

None declared.

\section{References}

[1] E.K. Blackburn, Primary capillary hemorrhage (including von Willebrand's disease), Brit J Haemat 7 (1961), $239-249$.

[2] E.C. Boerma, K.R. Mathura, P.H. van der Voort, P.E. Spronk, and C. Ince, Quantifying bedside-derived imaging of microcirculatory abnormalities in septic patients: A prospective validation study, Crit Care 9 (2005), R601-R606.

[3] A. Bollinger and B. Fagrell. Clinical Capillaroscopy: A Guide to Its Use in Clinical Research and Practice, Bern, Hogrefe \& Huber Publishers, Switzerland, 1990.

[4] A. Bollinger, A.J. Leu, U. Hoffmann and U.K. Franzeck, Microvascular changes in venous disease: An update, Angiology 48(1) (1997), 27-32.

[5] S.A. Conrad, P.T. Rycus and H. Dalton, Extracorporeal Life Support Registry Report 2004, Asaio J 51 (2005), 4-10.

[6] D. De Backer, J. Creteur, J.C. Preiser, M.J. Dubois and J.L. Vincent, Microvascular blood flow is altered in patients with sepsis, Am J Respir Crit Care Med 166 (2002), 98-104.

[7] D. De Backer, J. Creteur, M.J. Dubois, Y. Sakr and J.L. Vincent, Microvascular alterations in patients with acute severe heart failure and cardiogenic shock, Am Heart J 147 (2004), 91-99.

[8] D. De Backer, D.A. Ortiz and D. Salgado, Coupling microcirculation to systemic hemodynamics, Current Opinion in Critical Care 16 (2010), 001-005.

[9] A.M. Dondorp, C. Ince, P. Charunwatthana, J. Hanson, A. van Kuijen and M.A. Faiz et al., Direct in vivo assessment of microcirculatory dysfunction in severe falciparum malaria, J Infect Dis 197 (2008), 79-84.

[10] B. Fagrell, Vital microscopy: A clinical method for evaluating the risk of skin necrosis in patients with occlusive arterial disease, Bibl Anat 11 (1973), 328-333.

[11] S.K. Frazier and G.J. Skinner, Pulmonary artery catheters: State of the controversy, J Cardiovasc Nurs 23(2) (2008), 113-121.

[12] S.O.Freedlander and C.H. Lenhart, Clinical observations on the capillary circulation, Arch Intern Med 29 (1922), 12-32.

[13] P.T. Goedhart, M. Khalilzada, R. Bezemer, J. Merza and C. Ince, Sidestream Dark Field (SDF) imaging: A novel stroboscopic LED ring-based imaging modality for clinical assessment of the microcirculation, Opt Express 15 (2007), 15101-15114. 
[14] W. Groner, J.W. Winkelman, A.G. Harris, C. Ince, G.J. Bouma, K. Messmer and R.G. Nadeau, Orthogonal polarization spectral imaging: A new method for study of the microcirculation, Nat Med $\mathbf{5}$ (1999), 1209-1212.

[15] S.E. Hermansen, T. Naesheim, O.J. How and T. Myrmel, Circulatory assistance in acute heart failure-where do we go from here?, Scand Cardiovasc J 43 (2009), 211-216.

[16] S.M. Hollenberg, C.J. Kavinsky and J.E. Parrillo, Cardiogenic shock, Ann Intern Med 131 (1999), 47-59.

[17] C. Jung, M. Ferrari, R. Gradinger, M. Fritzenwanger, R. Pfeifer, M. Schlosser, T.C. Poerner, B.R. Brehm and H.R. Figulla, Evaluation of the microcirculation during extracorporeal membrane-oxygenation, Clin Hemorheol Microcirc 40 (2008), 311-314.

[18] F. Jung, From hemorheology to microcirculation and regenerative medicine: Fahraeus Lecture 2009, Clin Hemorheol Microcirc 45(2-4) (2010), 79-99.

[19] G.J. Magovern Jr. and K.A. Simpson, Extracorporeal membrane oxygenation for adult cardiac support: The Allegheny experience, Ann Thorac Surg 68 (1999), 655-661.

[20] Y. Kabasakal, D.M. Elvins, E.F. Ring and N.J. McHugh, Quantitative nailfold capillaroscopy findings in a population with connective tissue disease and in normal healthy controls, Ann Rheum Dis 55 (1996), 507-512.

[21] A. Krogh, The number and distribution of capillaries in muscle with the calculation of the oxygen pressure necessary for supplying the tissue, J Physiol 52 (1919), 409-515.

[22] J.K. Koscielny, R. Latza, S. Mürsdorf, C. Mrowietz, H. Kiesewetter, E. Wenzel, F. and Jung, Capillary microscopic and rheological dimensions for the diagnosis of von Willebrand disease in comparison to other haemorrhagic diatheses, Thromb Haemost 84(6) (2000), 981-988.

[23] C. Lam, K. Tyml, C. Martin and W. Sibbald, Microvascular perfusion is impaired in a rat model of normotensive sepsis, J Clin Invest 94 (1994), 2077-2083.

[24] C. Lehmann, V. Cerny, I. Abdo, H. Kern and M. Sander, Microcirculation diagnostics and applied studies in circulatory shock - research from the bench to the bedside, Clin Hemorheol Microcirc 52 (2012), 131-139.

[25] B. Leithäuser, C. Mrowietz, B. Hiebl, G. Pindur and F. Jung, Capillary bleeding under oral anticoagulation, Clin Hemorheol Microcirc 43(1) (2009), 167-171.

[26] P.D. Line, P. Mowinckel, B. Lien and K. Kvernebo, Repeated measurement variation and precision of laser Doppler flowmetry measurements, Microvasc Res 43 (1992), 285-293.

[27] J.M. Luce and G.D. Rubenfeld, Can Health Care Costs Be Reduced by Limiting Intensive Care at the End of Life? Am J Respir Crit Care Med 165 (2002), 750-754.

[28] C. Mork, K. Kvernebo, C.L. Asker and E.G. Salerud, Reduced skin capillary density during attacks of erythromelalgia implies arteriovenous shunting as pathogenetic mechanism, J Invest Dermatol 119 (2002), 949-953.

[29] G.E. Nilsson, T. Tenland and P.A. Oberg, Evaluation of a laser Doppler flowmeter for measurement of tissue blood flow, IEEE Trans Biomed Eng 27 (1980), 597-604.

[30] R.D. Piper, M. Pitt- Hyde, F. Li, W.J. Sibbald and R.F. Potter, Microcirculatory changes in rat skeletal muscle in sepsis, Am J Respir Crit Care Med 154 (1996), 931-937.

[31] C.A. den Uil, W.K. Lagrand, E. van der Ent, L.S.D. Jewbali, J.M. Cheng, P.E. Spronk and M.L. Simoons, Impaired microcirculation predicts poor outcome of patients with acute myocardial infarction complicated by cardiogenic shock, Eur Heart J 31 (2010), 3032-3039.

[32] T. Wester, E. Häggblad, Z.A. Awan, A. Barrat-Due, M. Kvernebo, P.S. Halvorsen, T.E. Mollnes and K. Kvernebo, Assessments of skin and tongue microcirculation reveals major changes in porcine sepsis, Clin Physiol Funct Imaging 31 (2011), $151-158$

[33] (www.oslo-universitetssykehus.no/omoss/avdelinger/thoraxkirurgisk/Sider/manualer.aspx (in Norwegian).

[34] K. Wårdell, A. Jakobsson and G.E. Nilsson, Laser Doppler perfusion imaging by dynamic lightscattering, IEEE Trans Biomed Eng 40(4) (1993), 309-16. 